WAPD-TM-1637

Detailed Analyses of Dynamic and Static Errors in Neutron Radiography Testing

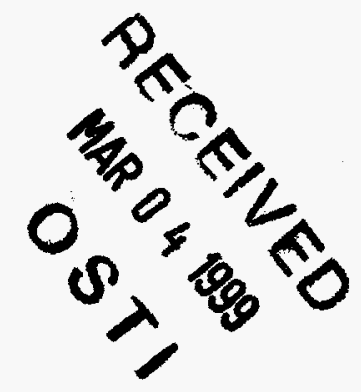

H. Joo

S. S. Glickstein

Bettis Atomic Power Laboratory

West Mifflin, Pennsylvania 15122

January 1999

DISTRIBUTION OF THIS DOCUMENT IS UNMMTTED ph

Prepared for the

U.S. Department of Energy

by Westinghouse Electric Company

a division of CBS Incorporated

Under Contract No. DE-AC11-93PN38195 


\section{DISCLAIMER}

This report was prepared as an account of work sponsored by an agency of the United States Government. Neither the United States Government dor any agency thereof, nor any of their employees, makes any wartanty, express or implied. or assumes any legal liability or responsibility for the accuracy, completeress, or usefulness of any information, apparatus, product, or process disclosed. or represents that its use would not infringe privately owned rights. Reference beren wo any specific commercial product, process, or service by trade name. tradernart manufac. turer, or otherwise does not necessarily constitute or imply its endorsement. recommendation. or favoring by the United States Government or any ageocy thereof. The views and opinions of authors expressed herein do not becessarily sate or reflect those of the United States Government or any agency thereof. 


\section{DISCLAIMER}

Portions of this document may be illegible in electronic image products. Images are produced from the best available original document. 


\title{
Detailed Analyses of Dynamic and Static Errors in Neutron Radiography Testing
}

\author{
H. Joo and S. S. Glickstein
}

\section{DE-AC11-93PN38195}

Printed in the United States of America

Available from the

National Technical Information Service

U.S. Department of Commerce 5285 Port Royal Road

Springfield, Virginia 22151

\section{NOTE}

This document is an interim memorandum prepared primarily for internal reference and does not represent a final expression of the opinion of Westinghouse. When this memorandum is distributed externally, it is with the express understanding that Westinghouse makes no representation as to completeness, accuracy, or usability of Information contained therein. 


\section{NOTICE}

This report was prepared as an account of work sponsored by the United States Government. Neither the United States, nor the United States Department of Energy, nor any of their employees, nor any of their contractors, subcontractors, or their employees, makes any warranty, express or implied, or assumes any legal llability or responsibillty for the accuracy, completeness or usefulness of any information, apparatus, product or process disclosed, or represents that its use would not infringe privately owned rights. 


\section{Detailed Analyses of Dynamic and Static Errors in Neutron Radiography Testing}

H. Joo and S. S. Glickstein

\section{TABLE OF CONTENTS}

\section{ABSTRACT}

I. INTRODUCTION $\ldots \ldots \ldots \ldots \ldots \ldots \ldots \ldots \ldots \ldots \ldots \ldots \ldots \ldots \ldots \ldots$

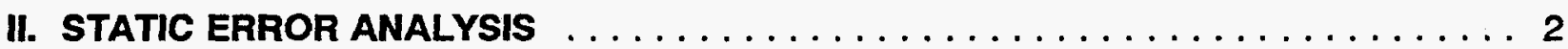

II.1 Exponential Attenuation Method $\ldots \ldots \ldots \ldots \ldots \ldots \ldots \ldots \ldots \ldots$

II.2 Static Error in Vapor Fraction Measurement $\ldots \ldots \ldots \ldots \ldots \ldots \ldots$

II.3 Maximum Static Error . . . . . . . . . . . . . . . . . . . . 6

III. DYNAMIC ERROR ANALYSIS $\ldots \ldots \ldots \ldots \ldots \ldots \ldots \ldots \ldots \ldots$

III.1 Exponential Attenuation Method $\ldots \ldots \ldots \ldots \ldots \ldots \ldots \ldots \ldots$

III.1.1 Binomial Vapor Fractions $\ldots \ldots \ldots \ldots \ldots \ldots \ldots \ldots \ldots \ldots \ldots \ldots$

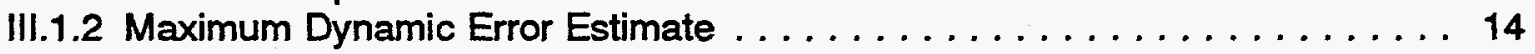

III.2 Collision Probability Method $\ldots \ldots \ldots \ldots \ldots \ldots \ldots \ldots \ldots \ldots \ldots$

III.3 Dynamic Error from Linear Vapor Fraction $\ldots \ldots \ldots \ldots \ldots \ldots \ldots \ldots$

III.3.1 Linear Vapor Fraction $\ldots \ldots \ldots \ldots \ldots \ldots \ldots \ldots \ldots \ldots \ldots \ldots .21$

III.3.2 Maximum Dynamic Error Estimate - Linear Vapor Fraction . . . . . . . . 22

III.3.3 Estimate of Dynamic Error for Acrylic Disc Measurements . . . . . . . . . . 24

IV. SUMMARY AND CONCLUSIONS $\ldots \ldots \ldots \ldots \ldots \ldots \ldots \ldots \ldots \ldots \ldots \ldots$

V. ACKNOWLEDGEMENT $\ldots \ldots \ldots \ldots \ldots \ldots \ldots \ldots \ldots \ldots \ldots \ldots \ldots \ldots$

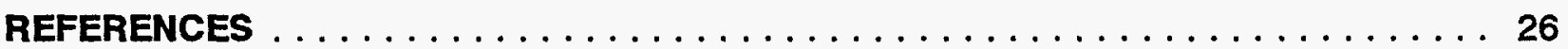


INTENTIONALLY BLANK 


\title{
Detailed Analyses of Dynamic and Static Errors in Neutron Radiography Testing
}

\author{
H. Joo and S. S. Glickstein \\ Westinghouse Electric Company \\ Bettis Atomic Power laboratory
}

\begin{abstract}
Neutron radiography systems are being used for real-time visualization of the dynamic behavior as well as time-averaged measurements of spatial vapor fraction distributions for two phase fluids. The extraction of quantitative data on vapor-liquid flow fields is a significant advance in the methodology of fundamental two-phase flow experimentation. The data in the form of video images are typically recorded on videotape at 30 frames per second. Image analysis of the video pictures is used to extract time-dependent or time-averaged data. The determination of the average vapor fraction requires averaging of the logarithm of time-dependent intensity measurements of the neutron beam (gray scale distribution of the image) that passes through the fluid. This could be significantly different than averaging the intensity of the transmitted beam and then taking the logarithm of that term. This is termed the dynamic error (error in the time-averaged vapor fractions due to the inherent time-dependence of the measured data) and is separate from the static error (statistical sampling uncertainty). The results provide insight into the characteristics of these errors and help to quantify achievable bounds on the limits of these errors. The static error was determined by the uncertainties of measured beam intensities. It was found that the maximum static error increases as liquid thickness increases and can be reduced by increasing the neutron source strength. The dynamic error increased with large fluctuations in the local vapor fractions and with increasing liquid thickness. Detailed analyses of both sources of errors are discussed.
\end{abstract}


INTENTIONALLY BLANK 


\section{INTRODUCTION}

As a unique aspect of our Fundamental Fluid Dynamics Development program, a neutron radiography system has been developed into a mature testing technique. It is being used for real-time visualization of dynamic behavior as well as time-averaged measurements of spatial vapor fraction distributions for two-phase flow in opaque conduits (References 1 through 4). While neutron radiography is an established technique for non-destructive testing, its use for real-time visualization and extraction of quantitative data on vapor-liquid flow fields is a significant advance in the methodology of fundamental two-phase flow experimentation.

A video-image analysis technique has been developed to extract average vapor fractions from real-time video-taped neutron attenuation data obtained at the Pennsylvania State University neutron radiography facility. This consists of measuring the gray level distribution throughout the flow region "illuminated" by the thermal neutron beam. In real-time television pictures, liquid regions appear dark (high neutron removal), vapor appears light, and neutron absorbing tracers appear black. Using a real-time neutron radiography camera, the turbulent motion of the boiling water is clearly visible through $1.5 \mathrm{~mm}$ stainless-steel conduit walls and $5 \mathrm{~cm}$ thick aluminum backup support plates placed on both sides of the conduit. The timedependent brightness information (gray level data) are processed to produce a spatial distribution of time-averaged vapor fraction over the illuminated region of the conduit. A data reduction system has been developed to handle the large amount of data that has been accumulated during a measurement period. The data reduction system was initially based on a time-averaging technique which can cause an error (dynamic error) that is related to fluctuations in the local density of the two-phase fluid. In a flow where there are no fluctuations in the fluid density during a unit counting interval, there still exist statistical uncertainties (static error) in measured variables. The main source of the static error is considered to be the neutron beam intensity and the gain of the neutron-image intensifier that converts a neutron image into a visible image. An in-depth analysis of both the static and dynamic errors was conducted to help quantify the achievable errors and also to provide insight into the systematic errors associated with the neutron radiography technique developed for vapor fraction measurements.

The potential static error in the vapor fraction resulting from a single-sample experiment is analytically estimated in this report by the second-power equation of the propagation of the uncertainties of individual variables (i.e., raw data). Also presented is the possible maximum uncertainty in the vapor fractions under specific operating conditions.

The dynamic error is related to the time-dependent fluctuations in the local composition of vapor-liquid mixtures. It results from the fact that the logarithm of the time-averaged intensity of the transmitted neutron beam ("Count-Mode Measure", Reference 5) is not equal to the time-averaged value of the logarithm of the intensity of the beam which is considered to be exact. In earlier tests, a few sets of neutron radiography data obtained for a rotating acrylic disc with holes (Reference 3) were processed by both the Count-Mode Measure technique and the Exact Measure technique. Negligible differences were found in the resulting void fractions between the two data processing techniques. However, to better understand the differences between these two methods of analyses an analytic evaluation of the dynamic error has been conducted in this report. An upper limit of possible maximum dynamic error is estimated for a test device of interest. 


\section{STATIC ERROR ANALYSIS}

When neutron radiography results are analyzed, some measure of the reliability of the results must be provided. From the point of view of reliability estimates, experiments fall into two overlapping categories - single-sample and multiple-sample experiments (Reference 6). Ideally, we would like to repeat all measurements enough times using enough observers and enough diverse instruments (i.e., multiple-sampling) so that the reliability of the results could be assured by the use of statistics. Unfortunately, in many experiments it is not practical to estimate all of the uncertainties of observation by repetition. Therefore, many experimenters rely on single-sample experiments in which uncertainties are not found by repetition.

The uncertainties in each of the basic variables involved in a single-sample experiment are described in this section to determine how these uncertainties propagate into the results. "Variable" will mean a basic quantity observed directly in the experiment as opposed to the "result" which is obtained by calculations with the recorded values of the variables. The maximum uncertainty for the result of a vapor fraction measurement, called the maximum static error, is determined.

\section{II.1 Exponential Attenuation Method}

While the neutron beam traverses a medium, its intensity attenuates due to scattering and absorption. An exponential attenuation can be formulated in terms of the attenuation coefficient and the thickness of each component of the medium. Consider a beam of neutrons impinging on a conduit containing a vapor-water mixture. If the change of the neutron intensity is approximated by an exponential attenuation, the vapor fraction $F(x, y)$ measured along the neutron beam direction at the point $(x, y)$ on the surface of the twophase flow regime is given (Reference 7 ) by

$$
F(x, y)=\frac{\ln \left[\frac{\ln \left[\frac{V(x, y)}{E(x, y)}\right]}{E(x, y)}\right]}{1-\frac{\mu_{v}}{\mu_{w}}},
$$

where

$$
\begin{aligned}
& V(x, y)=\text { the intensity of the transmitted beam at }(x, y) \text { when the conduit is filled with } \\
& \text { a mixture of water and vapor, } \\
& W(x, y)=\text { the beam intensity when the conduit is filled with water, } \\
& E(x, y)=\text { the beam intensity when the conduit is empty, } \\
& \mu_{v}=\text { the effective attenuation coefficient of vapor, and } \\
& \mu_{w} \quad=\text { the effective attenuation coefficient of water. }
\end{aligned}
$$

Equation (A-1) can be written as follows: 


$$
F(x, y)=C_{0} \frac{\ln \left[\frac{V}{W}\right]}{\ln \left[\frac{E}{W}\right]},
$$

where

$$
\mathrm{C}_{0}=\left[1-\frac{\mu_{v}}{\mu_{w}}\right]^{-1}
$$

The constant $C_{o}$ depends on the densities of vapor and water under a test condition. For a pressurized and heated two-phase flow system of interest, the constant $C_{0}$ ranges from unity to approximately 1.2 .

\section{II.2 Static Error in Vapor Fraction Measurement}

Since the vapor fraction in Equation (A-2) is a function of the three measured variables, $V(x, y), W(x, y)$ and $E(x, y)$, the relation between the uncertainties of the variables and the uncertainty for the result, $F(x, y)$, can be calculated by the second-power equation of the propagation of errors (References 6 and 8 ). The constant $C_{0}$ is assumed to be independent of these variables.

Applying the propagation of errors to Equation (A-2) gives

$$
(\Delta F)^{2}=\left(\frac{\partial F}{\partial V}\right)^{2}(\Delta V)^{2}+\left(\frac{\partial F}{\partial W}\right)^{2}(\Delta W)^{2}+\left(\frac{\partial F}{\partial E}\right)^{2}(\Delta E)^{2} .
$$

Dividing through by $F^{2}$, we obtain

$$
\left(\frac{\Delta F}{F}\right)^{2}=\left(\frac{\partial F}{\partial V}\right)^{2}\left(\frac{\Delta V}{F}\right)^{2}+\left(\frac{\partial F}{\partial W}\right)^{2}\left(\frac{\Delta W}{F}\right)^{2}+\left(\frac{\partial F}{\partial E}\right)^{2}\left(\frac{\Delta E}{F}\right)^{2} .
$$

From Equation (A-2), we can determine derivatives:

$$
\frac{\partial F}{\partial V}=\frac{C_{0}}{V \ln \left[\frac{E}{W}\right]},
$$$$
\frac{\partial F}{\partial W}=\frac{C_{0} \ln \left[\frac{V}{E}\right]}{W\left(\ln \left[\frac{E}{W}\right]\right]^{2}} \text {, and }
$$

$$
\frac{\partial F}{\partial E}=\frac{-C_{0} \ln \left[\frac{V}{W}\right]}{E\left(\ln \left[\frac{E}{W}\right]^{2}\right.} \text {. }
$$


Substituting these three derivatives into Equation (A-3) yields

$$
\left(\frac{\Delta F}{F}\right)^{2}=\frac{C_{0}^{2}}{F^{2}\left(\ln \left[\frac{E}{W}\right]\right]^{2}}\left\{\left(\frac{\Delta V}{V}\right)^{2}+\left(\frac{\ln \left[\frac{V}{E}\right]}{\ln \left[\frac{E}{W}\right]}\right)^{2}\left(\frac{\Delta W}{W}\right)^{2}+\left(\frac{\ln \left[\frac{V}{W}\right]}{\ln \left[\frac{E}{W}\right]}\right)^{2}\left(\frac{\Delta E}{E}\right)^{2}\right\}
$$

If we assume that the inherent statistical uncertainty of the neutron beam intensity is independent of the medium in the test section, then we can approximate

$$
\frac{\Delta V}{V} \approx \frac{\Delta W}{W} \approx \frac{\Delta E}{E}
$$

From Equation (A-2)

$$
\frac{\ln \left[\frac{V}{E}\right]}{\ln \left[\frac{E}{W}\right]}=\frac{F}{C_{0}}-1
$$

Using Equations (A-5) and (A-6), Equation (A-4) yields

$$
\begin{aligned}
\left(\frac{\Delta F}{F}\right)^{2} & =\frac{C_{0}^{2}\left(\frac{\Delta V}{V}\right)^{2}}{F^{2}\left(\ln \frac{E}{W}\right)^{2}}\left\{1+\left(\frac{F}{C_{0}}-1\right)^{2}+\left(\frac{F}{C_{0}}\right)^{2}\right\} \\
& =\frac{\left(\frac{\Delta V}{V}\right)^{2}}{\bar{F}^{2}\left\{\ln \frac{E}{W}\right)^{2}}\left\{1+(\bar{F}-1)^{2}+\bar{F}^{2}\right\},
\end{aligned}
$$

where $\bar{F}=F / C_{0}$.

Therefore, we have a relative error of $F(x, y)$ as follows:

$$
\frac{\Delta F(x, y)}{F(x, y)}=\frac{\left(\frac{\Delta V}{V}\right) \sqrt{2\left(1-\bar{F}+\bar{F}^{2}\right)}}{\dot{F} \ln \left[\frac{E(x, y)}{W(x, y)}\right]} .
$$

From Reference 7, we have

$$
\ln \left[\frac{E(x, y)}{W(x, y)}\right]=\mu_{w} T(x, y)
$$


where $T(x, y)$ is the flow path thickness at a point $(x, y)$. Rewriting Equation (A-7) gives

$$
\frac{\Delta F(x, y)}{F(x, y)}=\frac{\sqrt{2} \frac{\Delta V(x, y)}{V(x, y)}}{\mu_{w} T(x, y)} \sqrt{1-\frac{1}{\bar{F}}+\frac{1}{\bar{F}^{2}}} .
$$

In Eq. (A-8), the term $\triangle V N$ is the inherent statistical uncertainty of the intensity of the transmitted neutron beam at $(x, y)$ when the conduit is filled with a mixture of water and vapor.

We can have some estimate of the sample variance if we were to repeat the measurement many times. Because we have only a single measurement, however, the sample variance cannot be calculated directly but must be estimated by analogy with an appropriate statistical model. If we make the assumption that the measurement has been drawn from a population whose theoretical distribution function is predicted by a statistical model, then we must match an appropriate theoretical distribution to the available data. In a neutron beam experiment, the neutron population measured can be represented by a Poisson distribution function (References 8 and 9). In the Poisson distribution model, the best estimate of the deviation from the mean (i.e., one value of the standard deviation) that typify our single measurement is equal to the square root of the measurement (References 6 and 8). Let us assume that detection of the transmitted neutrons is characterized by a Poisson process (References 8 and 9 ), which implies that

$$
\begin{aligned}
\frac{\Delta V(x, y)}{V(x, y)} & =\frac{\sqrt{N(x, y)}}{N(x, y)} \\
& =\frac{1}{\sqrt{N(x, y)}},
\end{aligned}
$$

where

$$
\begin{aligned}
& N(x, y)=\begin{array}{l}
\text { number of counts measured by a unit detector element corresponding to a } \\
\text { position }(x, y) \text { in the test device. }
\end{array}
\end{aligned}
$$

When a real-time video camera is used to record the intensity of transmitted neutrons, the final unit detector element is a small surface area which corresponds to a single video image pixel of the camera.

In converting the detectable neutron intensity to a measurable signal, the number of counts at a steady-state condition measured during a unit counting interval is given by

$$
N(x, y)=G_{0} t_{0} \Delta x \Delta y V(x, y)
$$

where

$$
\begin{aligned}
& \mathbf{G}_{\theta} \quad=\begin{array}{l}
\text { converter gain in signal intensity } \\
=
\end{array} \\
& \text { number of counts of measurable signal per neutron interacting with the } \\
& \text { detector (counts/neutrons), } \\
& t_{0} \quad \text { counting interval }(\mathrm{sec}), \\
& \Delta x \Delta y=\text { surface area of unit detector element }\left(\mathrm{cm}^{2}\right), \text { and } \\
& \left.V(x, y)=\text { intensity of transmitted neutron beam (neutrons } / \mathrm{cm}^{2} \mathrm{sec}\right) .
\end{aligned}
$$


A maximum inherent static error is evaluated based on Eq. (A-8) in the following section for the Penn State neutron radiography facility which has a thermal neutron flux level on the order of $2 \times 10^{6} \mathrm{n} / \mathrm{cm}^{2} / \mathrm{s}$ at 1 megawatt reactor power.

\section{II.3 Maximum Static Error}

Combining Equation (A-8) and Equation (A-9) produces an expression of an absolute magnitude of the static error as follows:

$$
\begin{aligned}
\Delta F(x, y) & =\frac{\sqrt{2}}{\mu_{W} T(x, y)} \frac{F(x, y)}{\sqrt{N(x, y)}} \sqrt{1-\frac{1}{\bar{F}}+\frac{1}{\bar{F}^{2}}} \\
& =\frac{\sqrt{2}}{\mu_{W} T(x, y)} \frac{1}{\sqrt{N(x, y)}} \sqrt{F^{2}-C_{0} F+C_{0}^{2}} .
\end{aligned}
$$

Given a test section dimension about $\mu_{w} T(x, y)$ and information on the neutron source strength and detector characteristics that define $N(x, y)$ in Equation (A-10), we can find the maximum possible error $\Delta F(x, y)$ examining the behavior of the last two terms in Equation (A-11).

From Reference 7, we have the transmitted beam intensity that is recorded by the detector:

$$
V(x, y)=E(x, y) e^{-R(x, y)\left[1-F(x, y) / \kappa_{0}\right]},
$$

where $R(x, y)=\mu_{w} T(x, y)$.

Then the number of counts defined by Equation $(A-10)$ becomes

$$
\begin{aligned}
N(x, y) & =G_{0} t_{0} \Delta x \Delta y E(x, y) e^{-R\left[1-F K_{0}\right]} \\
& =N_{0}(x, y) e^{-R\left[1-F K_{0}\right]},
\end{aligned}
$$

where $N_{0}(x, y)=G_{0} t_{0} \Delta x \Delta y E(x, y)$ and it is a constant independent of the two-phase fluid. $N_{o}(x, y)$ is solely dependent upon the detector specification including the background intensity of the transmitted beam, $E(x, y)$.

The static error in Equation (A-11) is rewritten as follows:

$$
\Delta F(x, y)=\sqrt{\frac{2}{N_{0}(x, y)}} \frac{\sqrt{F^{2}-C_{0} F+C_{0}^{2}}}{R(x, y)} e^{\left[1-F / C_{0}\right] R(x, y) / 2} .
$$

Equation (A-14) reveals that the static error in the vapor fraction is a function of three properties of a two-phase fluid system; $C_{0}, F(x, y)$ and $R(x, y)$.

Of interest is the maximum value of the static error given by Equation (A-14), for a test device specified in terms of $R(x, y)$. Let us find a vapor fraction $F(x, y)$ at which Equation $(A-14)$ results in the largest $\Delta F(x, y)$ by examining the derivative of Equation $(A-14)$ taken with respect to $F(x, y)$. 


$$
\frac{\partial \Delta F(x, y)}{\partial F(x, y)}=\sqrt{\frac{2}{N_{0}}} \frac{1}{2 R}\left\{\frac{2 F-C_{0}}{\sqrt{F^{2}-C_{0} F+C_{0}^{2}}}-\frac{R}{C_{0}} \sqrt{F^{2}-C_{0} F+C_{0}^{2}}\right\} e^{\left(1-F / C_{\partial}\right) R / 2}
$$

Let us find a vapor fraction $F_{m}(x, y)$ at which the above derivative becomes zero. Since the exponential term in Equation (A-15) is greater than zero, we have a condition

$$
2 F_{m}-C_{0}-\frac{R}{C_{0}}\left[F_{m}^{2}-C_{0} F_{m}+C_{0}^{2}\right]=0 \text {. }
$$

This condition results in a solution for $F_{m}(x, y)$ :

$$
F_{m}=\frac{C_{0}}{2}\left[\frac{2+R}{R}-\frac{\sqrt{4-3 R^{2}}}{R}\right] \text {. }
$$

This indicates that for $F_{m}(x, y)$ to be a real number $R(x, y)$ must not be greater than $2 / \sqrt{3}$. The plus sign of the square root term was deleted in Equation (A-17) to eliminate meaningless vapor fractions greater than unity.

Substituting $F_{m}(x, y)$ in Equation (A-17) into Equation (A-14) provides

$$
\left.\Delta F(x, y)\right|_{F=F_{m}}=\sqrt{\frac{2}{N_{0}}} \frac{C_{0}}{R^{2}} \frac{2 F_{m}-C_{0}}{\sqrt{F_{m}^{2}-C_{0} F+C_{0}^{2}}} e^{\left(1-F_{m} / C_{\alpha}\right) R / 2} .
$$

Let us evaluate $\Delta F(x, y)$ at the limiting values of $F(x, y)$, zero and unity, and compare the resulting $\Delta F(x, y)$ with Equation $(A-18)$ to find the largest of these $\Delta F(x, y)$ values.

$$
\begin{aligned}
\left.\Delta F(x, y)\right|_{F=0.0} & =\sqrt{\frac{2}{N_{0}}} \frac{C_{0}}{R} e^{R / 2} \\
\left.\Delta F(x, y)\right|_{F=1.0} & =\sqrt{\frac{2}{N_{0}}} \frac{\sqrt{1-C_{0}+C_{0}^{2}}}{R} e^{\left(1-1 / C_{0}\right) R / 2} \\
& =\sqrt{\frac{2}{N_{0}}} \frac{C_{0}}{R} e^{R / 2} \sqrt{1-\frac{1}{C_{0}}+\frac{1}{C_{0}^{2}}} e^{-R / 2 C_{0}}
\end{aligned}
$$

Since each of the last two factors in Equation (A-20) is not greater than unity, Equation (A-19) is always greater than or equal to Equation (A-20) (The values of $C_{0}$ range from 1.0 to 1.2). Let us now compare Equation (A-18) with Equation (A-19). Dividing Equation (A-18) by Equation (A-19) results in the following ratio:

$$
\begin{aligned}
\text { ratio } & =\frac{2 F_{m}-C_{0}}{R \sqrt{F_{m}^{2}-C_{0} F_{m}+C_{0}^{2}}} e^{-F_{m} R / 2 C_{0}} \\
& =\sqrt{1-\frac{F_{m}}{C_{0}}\left(1-\frac{F_{m}}{C_{0}}\right)} e^{-F_{m} R / 2 C_{0}}
\end{aligned}
$$


where the relationship in Equation (A-16) has been introduced. Since (a) the vapor fraction $F_{m}(x, y)$ cannot become greater than unity, (b) the minimum value of $C_{0}$ is unity, and (c) the exponential term is smaller than unity, the above ratio is less than unity. Therefore, it is concluded that the maximum static error in vapor fraction occurs when the vapor fraction reaches its lowest value, zero. From Equation (A19)

$$
\operatorname{Max} \Delta F(x, y)=\sqrt{\frac{2}{N_{0}(x, y)}} \frac{C_{0}}{R(x, y)} e^{R(x, y) / 2} \text {. }
$$

For the neutron radiography facility at Penn State, we have typical specifications for $N_{0}(x, y)$ defined by Equation $(A-13)$ :

Gain; $G_{\mathbf{e}} \approx 300$ counts/neutron (References 10 and 11)

Counting interval; $t_{0}=1 / 30 \mathrm{sec}$ (for a single frame of real-time video image) Detector element size; $\Delta x=\Delta y=0.05 \mathrm{~cm}$ (corresponding to a video pixel)

Source neutron intensity; $I_{0}=2 \times 10^{6}$ neutrons $/ \mathrm{cm}^{2} \cdot \mathrm{sec}$

While Reference 10 indicates the gain to be approximately 1,000 , we have used a more conservative figure of 300 as indicated in Reference 11.

The neutron intensity of transmitted beam incident upon the detector-converter system, $E(x, y)$, is reduced from the intensity of the source beam, $I_{0}$. Assuming a reduction of the transmitted beam to $50 \%$ of the source, we have the number of counts $N_{0}(x, y)$ calculated as follows:

$$
\begin{aligned}
N_{0}(x, y) & =G_{0} t_{0} \Delta x \Delta y E(x, y) \\
& =300 \cdot(1 / 30) \cdot\left(0.05^{2}\right) \cdot\left(2 \times 10^{6} \times 0.5\right) \text { counts } \\
& =2.5 \times 10^{4} \text { counts. }
\end{aligned}
$$

The worst-case maximum static error in vapor fractions is evaluated as a function of the relative path thickness, $R(x, y)$, using the limiting value of $C_{0}=1.2$. Results are shown in Table 1 and Figure 1. The maximum static error occurs when the vapor fraction is zero, Equation (A-19). Also shown in Figure 1 is the static error when the vapor fraction is unity, Equation (A-20). 
Table 1. Maximum Static Error versus Path Thickness

\begin{tabular}{|c|c|c|}
\hline$R(x, y)^{*}$ & $T(x, y)(c m)$ & $M a x . \Delta F(x, y)$ \\
\hline 0.1 & 0.033 & 0.113 \\
\hline 0.2 & 0.067 & 0.059 \\
\hline 0.5 & 0.167 & 0.028 \\
\hline 0.66 & 0.220 & 0.023 \\
\hline 1.0 & 0.333 & 0.018 \\
\hline 2.0 & 0.667 & 0.015 \\
\hline 3.0 & 1.000 & 0.016 \\
\hline 4.0 & 1.333 & 0.020 \\
\hline 5.0 & 1.667 & 0.026 \\
\hline 7.0 & 2.333 & 0.051 \\
\hline 9.0 & 3.000 & 0.107 \\
\hline Based on the attenuation coefficient of water of $3.0 \mathrm{~cm}^{-1}$ \\
\hline
\end{tabular}

For a test device having $\mu_{w}=3.0 \mathrm{~cm}^{-1}$ and $T(x, y)=0.22 \mathrm{~cm}$ or $\mathrm{R}(x, y)=0.66$, the maximum static error is 0.023 in the measured vapor fraction. This error is the upper limit in the local vapor fraction of any magnitude from zero to unity. It is observed that a relatively small static error of up to 0.02 in vapor fractions when test devices of wide range of path thicknesses ranging from $R(x, y)=0.9$ to $R(x, y)=4.0$ (corresponding to $0.3 \mathrm{~cm}-1.3 \mathrm{~cm}$ in path thickness) are tested.

\section{DYNAMIC ERROR ANALYSIS}

A dynamic error analysis of vapor fraction measurement using neutron radiography is described in this section. The dynamic error is related to time-dependent fluctuations in the local density of a two-phase fluid. It results from the fact that the time-averaged value of the logarithm of the intensity of the transmitted neutron beam ("Exact Measure") is not equal to the logarithm of the time-averaged intensity of the beam ("Count-Mode Measure"), Reference 5. Since the Count-Mode has been used in the vapor fraction measurement in the past, it is necessary to discuss the dynamic error involved with the vapor fractions measured by the neutron radiography technique. The exponential attenuation method is analyzed first in this section for a limiting case represented by a binomial vapor fraction model. The Collision Probability Method (Reference 7) is then analyzed for the same case. Finally, a less limiting case in which the time-dependent vapor fraction is modeled in a linear function of time is analyzed. An upper limit of possible maximum dynamic error is determined in each case as a function of the two-phase medium thickness.

\section{III.1 Exponential Attenuation Method}

When a time-averaged vapor fraction is calculated, a time-averaged value of $V(x, y)$ in Eq. (A-2) is frequently used. Therefore, it is necessary to evaluate the dynamic error associated with the neutron radiography data processing technique that utilizes the time-averaged $V(x, y)$. The dynamic error results from the fact that the timeaveraged value of the logarithm, In $V(x, y)$, in Equation (A-2) is not equal to the logarithm of the time-averaged intensity of the transmitted beam, $V(x, y)$. 
The time-averaged vapor fraction calculated by the Count-Mode Measure with a time-averaged value of $V(x, y), \bar{V}(x, y)$, is given by

$$
\bar{F}_{c}(x, y)=c_{0} \frac{\ln \left[\frac{\bar{V}(x, y)}{W(x, y)}\right]}{\ln \left[\frac{E(x, y)}{W(x, y)}\right]},
$$

where $\bar{V}(x, y)=\frac{1}{T} \int_{0}^{T} V(x, y, t) d t, T=$ measurement time period, and

other quantities, $C_{0}, W(x, y)$ and $E(x, y)$, are time-independent.

Defining time-independent terms

$$
C_{1}=\frac{C_{0}}{\ln \left[\frac{E}{W}\right]} \quad \text { and } C_{2}=\frac{-C_{0} \ln W}{\ln \left[\frac{E}{W}\right]}
$$

we can rewrite Eq. (B-1) as follows:

$$
\overline{F_{c}}(x, y)=c_{1} \ln \bar{V}(x, y)+c_{2} \text {. }
$$

Let us consider a time period of $T$ during which an average vapor fraction is to be determined. The time period $\mathrm{T}$ is divided into $\mathrm{n}$ subintervals of equal length of $\Delta t$, and the value of $V(x, y, t)$ is assumed constant during $\Delta t$ from $t_{j}$ to $t_{j}+\Delta t$. We express $\bar{v}(x, y)$ as follows:

$$
\bar{V}(x, y)=\frac{1}{n} \sum_{j=1}^{n} v_{i}(x, y)
$$

where $V_{j}(x, y)=V(x, y, t)$ for $t_{j} \leq t<t_{j}+\Delta t$

Substituting Eq. (B-3) into Eq. (B-2) yields

$$
\bar{F}_{c}(x, y)=C_{1} \ln \left[\frac{1}{n} \sum_{j=1}^{n} V_{i}(x, y)\right]+c_{2} \text {. }
$$

Now, let us determine exact time-averaged vapor fraction $\bar{F}(x, y)$ applying a timeaveraged value of the logarithm of $V(x, y)$, i.e., Exact Measure, to Equation (A-2). 


$$
\begin{aligned}
\bar{F}(x, y) & =\frac{1}{T} \int_{0}^{T} F(x, y, t) d t \\
& =\frac{1}{T} \int_{0}^{T}\left[C_{1} \ln V(x, y, t)+C_{2}\right] d t \\
& =C_{1} \frac{1}{T} \int_{0}^{T} \ln V(x, y, t) d t+\frac{1}{T} \int_{0}^{T} C_{2} d t \\
& =C_{1} \frac{1}{n} \sum_{j=1}^{n}\left[\ln V_{i}(x, y)\right]+C_{2} \\
& =C_{1} \ln \left[\prod_{j=1}^{n} V_{i}(x, y)\right]^{1 / n}+C_{2} .
\end{aligned}
$$

The dynamic error $[\Delta \bar{F}(x, y)]$ is then calculated by subtracting Eq. (B-5) from Eq. (B-4), the result being,

$$
\begin{aligned}
\Delta \bar{F}(x, y) & =\bar{F}_{c}(x, y)-\bar{F}(x, y) \\
& =C_{1}\left\{\ln \left[\frac{1}{n} \sum_{j=1}^{n} v_{j}(x, y)\right]-\ln \left[\prod_{j=1}^{n} v_{i}(x, y)\right]^{1 / n}\right\} .
\end{aligned}
$$

Eq. $(B-6)$ indicates that whenever the intensity of the transmitted beam at $(x, y)$ during a time interval of $t_{j} \sim t_{i}+\Delta t, V_{j}(x, y)$, is known for every time subinterval, the dynamic error can be determined by the difference of the logarithms of the two types of the average values, $\frac{1}{n} \sum_{j=1}^{n} V_{j}(x, y)$ and $\left[\prod_{j=1}^{n} V_{j}(x, y)\right]^{1 / n}$.

\section{III.1.1 Binomial Vapor Fractions}

We are particularly interested in time-averaged vapor fractions of a two phase flow simulated by an acrylic disc with holes (partial or full depth) on it. This is applicable to experiments discussed in Reference 3. The timedependent vapor (void) fractions are represented as a series of step changes between two values of $F(x, y, t), F_{1}(x, y)$ and $F_{2}(x, y)$; that is a binomial pattern. Let $\delta(x, y)$ represent the difference between the values of $F_{1}(x, y)$ and $F_{2}(x, y)$.

Consider a case where $F_{2}(x, y)>F_{1}(x, y)$ and $F_{2}(x, y)=F_{1}(x, y)+\delta(x, y)$. Also defined are the intensities of the transmitted beam $V_{1}(x, y)$ and $V_{2}(x, y)$ corresponding to the vapor fractions $F_{1}(x, y)$ and $F_{2}(x, y)$, respectively.

During a time period of $T$, it is assumed that $V_{1}(x, y)$ is recorded at $m$ subintervals and $V_{2}(x, y)$ is recorded (or measured) at $(n-m)$ subintervals. It is also assumed that the time subinterval is sufficiently small so that the fluctuation in the measurement $V(x, y)$ within the subinterval is considered negligible. A fraction of the time period is defined as

$$
\alpha=m / n \text { or } 1-\alpha=(n-m) / n \text {. }
$$


Let us evaluate two logarithm terms in Eq. (B-6) for this binomial vapoi fraction case.

$$
\begin{aligned}
\frac{1}{n} \sum_{j=1}^{n} v_{j}(x, y) & =\frac{1}{n}\left[m v_{1}(x, y)+(n-m) v_{2}(x, y)\right] \\
& =\alpha v_{1}+(1-\alpha) v_{2} . \\
{\left[\prod_{j=1}^{n} v_{j}(x, y)\right]^{1 / n} } & =\left[v_{1}^{m}(x, y) v_{2}^{n-m}(x, y)\right]^{1 / n} \\
& =v_{1}^{\alpha} v_{2}^{1-\alpha} .
\end{aligned}
$$

Using the above relationships, Eq. (B-6) gives

$$
\Delta \bar{F}(x, y)=C_{1}\left\{\ln \left[\alpha V_{1}+(1-\alpha) V_{2}\right]-\alpha \ln V_{1}-(1-\alpha) \ln V_{2}\right\}
$$

Using $C_{1}$ and $C_{2}$ defined earlier, we have from Eq. (A-2) two relationships:

$$
\begin{aligned}
& V_{1}(x, y)=e^{-\left[C_{2}-F_{1}(x, y) y c_{1}\right.} \\
& V_{2}(x, y)=e^{-\left[C_{2}-F_{2}(x, y) y c_{1}\right.}
\end{aligned}
$$

Using the relationships given by Eqs. (B-8) and (B-9), Eq. (B-7) becomes

$$
\begin{gathered}
\Delta \bar{F}(x, y) / C_{1}=\ln \left\{\alpha e^{-\left(C_{2}-F_{1}\right) / C_{1}}+(1-\alpha) e^{-\left(C_{2}-F_{2}\right) / C_{1}}\right\} \\
+\alpha\left(C_{2}-F_{1}\right) / C_{1}+(1-\alpha)\left(C_{2}-F_{2}\right) / C_{1} .
\end{gathered}
$$

Or,

$$
\begin{aligned}
\Delta \bar{F}(x, y)= & c_{1} \ln \left\{\alpha e^{-c_{2} c_{1}} e^{F_{1} c_{1}}+(1-\alpha) e^{-c_{2} c_{1}} e^{F_{2} c_{1}}\right\} \\
& +\alpha C_{2}-\alpha F_{1}+(1-\alpha) c_{2}-(1-\alpha) F_{2} \\
= & c_{1} \operatorname{lin}\left\{e^{-c_{2} c_{1}}\left[\alpha e^{F_{1} c_{1}}+(1-\alpha) e^{F_{2} c_{1}}\right\}+\right. \\
& c_{2}-\alpha F_{1}-(1-\alpha) F_{2} \\
= & c_{1} \ln \left[\alpha e^{F_{1} c_{1}}+(1-\alpha) e^{\left(F_{1}+\delta\right) / c_{1}}\right] \\
& -\alpha F_{1}-(1-\alpha)\left(F_{1}+\delta\right)
\end{aligned}
$$

where the identity $F_{2}(x, y)=F_{1}(x, y)+\delta(x, y)$ has been used. 


$$
\text { Since } \begin{aligned}
C_{1} & =\frac{C_{0}}{\ln \left[\frac{E(x, y)}{W(x, y)}\right]} \text {, we can have } \\
\frac{C_{0}}{C_{1}} & =\ln E(x, y)-\ln W(x, y) \\
& =\ln E(x, y)-\ln \left[E(x, y) \cdot \exp \left\{-\mu_{w} T(x, y)\right\}\right] \\
& =\mu_{w} T(x, y),
\end{aligned}
$$

where a relationship between $E(x, y)$ and $W(x, y)$ shown in Reference 7 has been applied to. Therefore,

$$
C_{1}=\frac{C_{0}}{\mu_{w} T(x, y)} \text { or } \frac{1}{C_{1}}=\left[1-\frac{\mu_{v}}{\mu_{w}}\right] \mu_{W} T(x, y) .
$$

This indicates that $1 / C_{1}$ is the number of mean free paths of the conduit filled with water multiplied by the factor of $1-\mu_{w} / \mu_{W}$, which is unity for the acrylic disc with holes. However, for a pressurized hot conduit, this factor should be included. Finally, we have for acrylic discs with holes

$$
\begin{aligned}
\Delta \bar{F}(x, y)= & \frac{1}{\mu_{W} T(x, y)} \ln \left\{\alpha e^{\mu_{w} T(x, y) F_{1}(x, y)}+(1-\alpha) e^{\mu_{W} T(x, y)\left[F_{1}(x, y)+\delta(x, y)\right\}}\right\} \\
& -\alpha F_{1}(x, y)-(1-\alpha)\left[F_{1}(x, y)+\delta(x, y)\right] .
\end{aligned}
$$

Equation (B-12) can be used to estimate the dynamic error in void (not vapor) fraction measurement given the geometric (path thickness $T(x, y)$ ) and material (effective attenuation coefficient $\mu_{w}$ ) parameters of an acrylic disc with holes or equivalent test devices. Figure 2 presents the magnitude of the dynamic errors for an acrylic disc with holes (References 3 ) determined at various values of $\delta(x, y)$ and $\alpha$. The maximum possible dynamic error can be as large as 0.08 in void fractions, which is significantly larger than what was observed experimentally during the data acquisition process using the Exact Measure technique. This difference is discussed in Section III.3.3.

For a pressurized hot fluid $\mu_{v}$ is non-negligible. In this case, $\mu_{w}$ in Equation (B-12) must be replaced by $\left(\mu_{w}-\mu_{v}\right)$. For some cases of high pressures and high temperatures, the dynamic error may be reduced by up to 15 percent compared to conditions at standard pressure and temperature. (See Section III.1.2(f) and Figure 3.)

To get an idea of the phenomenon Eq. (B-12) represents, it is useful to see how it changes with each of the variables. The ranges of these variables are:

$$
\begin{aligned}
& \mu_{w} T(x, y)>0 \\
& 0.0 \leq \alpha \leq 1.0 \\
& 0.0 \leq F_{1}(x, y) \leq 1.0 \\
& 0.0 \leq \delta(x, y) \leq 1.0-F_{1}(x, y)
\end{aligned}
$$

path thickness

time fraction for $F_{1}(x, y)$

minimum vapor fraction

fluctuation in vapor fractions

It should be noted that the dynamic error equals zero when 
$\delta(x, y)=0.0$, that is $F_{2}(x, y)=F_{1}(x, y)$; or

$\alpha=0.0$ or $\alpha=1.0$,

which reduces the dynamic flow to the static case.

\section{III.1.2 Maximum Dynamic Error Estimate}

To find out how each variable in Eq. (B-12) affect the dynamic error, the derivatives of the equation taken with respect to each variable are examined in this section (Reference 5). A maximum possible dynamic error is determined thereafter.

(a) Change with ew I( $x, y)$

Defining $R(x, y)=\mu_{w} T(x, y)$ and taking the derivative of Eq. (B-12) with respect to $R(x, y)$ give

$$
\begin{aligned}
\frac{\partial \Delta \bar{F}(x, y)}{\partial R(x, y)}= & \frac{-1}{R^{2}} \ln \left\{\alpha e^{R F_{1}}+(1-\alpha) e^{R\left[F_{1}+\delta\right]}\right\} \\
& +\frac{1}{R} \frac{\alpha F_{1} e^{R F_{1}}+(1-\alpha)\left[F_{1}+\delta\right] e^{\left.R F F_{1}+\delta\right]}}{\alpha e^{R F_{1}}+(1-\alpha) e^{R\left[F_{1}+\delta\right]}} \\
= & -\frac{1}{R^{2}}\left[R F_{1}+\ln \left\{\alpha+(1-\alpha) e^{R \delta}\right\}\right] \\
& +\frac{1}{R}\left[\frac{\alpha F_{1}+(1-\alpha)\left[F_{1}+\delta\right] e^{R \delta}}{\alpha+(1-\alpha) e^{R \delta}}\right] . \\
\frac{\partial \Delta \bar{F}(x, y)}{\partial R(x, y)}= & \frac{1}{R^{2}}\left[\frac{(1-\alpha) R \delta e^{R \delta}}{\left.\alpha+(1-\alpha) e^{R \delta}-\ln \left\{\alpha+(1-\alpha) e^{R \delta}\right\}\right] .}\right.
\end{aligned}
$$

A numerical evaluation indicates that $\partial \Delta \bar{F} / \partial R$ is greater than zero for variables within the range of interest as shown in Table 2.

Since $R(x, y)=\mu_{w} T(x, y)$ and $\mu_{w}$ depends on water (or acrylic disc) density and neutron energy spectrum, which are constant during a measurement, we would expect an increase in the error with an increase in the thickness of the test section.

Table 2. Change of Dynamic Error with $R(x, y)$

\begin{tabular}{|c|c|c|}
\hline$R(x, y)$ & $\begin{array}{c}\text { Eq. (B-13) } \\
\delta=0.5, \alpha=0.5\end{array}$ & $\begin{array}{c}\text { Eq. (B-13) } \\
\delta=0.8, \alpha=0.5\end{array}$ \\
\hline 0.1 & 0.0312 & 0.0799 \\
\hline 0.5 & 0.0310 & 0.0784 \\
\hline 1.0 & 0.0303 & 0.0740 \\
\hline 2.0 & 0.0277 & 0.0601 \\
\hline
\end{tabular}


(b) Change with $\alpha$

Using Eq. (B-12), the value of $\partial \Delta \bar{F}(x, y) / \partial \alpha$ is

$$
\begin{aligned}
& \frac{\partial \Delta \bar{F}(x, y)}{\partial \alpha}=\frac{1}{R} \frac{e^{R F_{1}}-e^{R\left[F_{1}+\delta\right]}}{\alpha e^{R F_{1}}+(1-\alpha) e^{R\left[F_{1}+\delta\right]}}-F_{1}+\left[F_{1}+\delta\right] . \\
& \frac{\partial \Delta \bar{F}(x, y)}{\partial \alpha}=\frac{1-e^{A \delta}}{R\left[\alpha+(1-\alpha) e^{R \delta}\right]}+\delta .
\end{aligned}
$$

The point of maximum error $\left(\alpha=\alpha_{m}\right)$ is where the value of $\partial \Delta \bar{F} / \partial \alpha=0$. Hence,

$$
0=\frac{1-e^{R \delta}}{R\left[\alpha_{m}+\left(1-\alpha_{m}\right) e^{R \delta}\right]}+\delta .
$$

Rearranging gives an $\alpha$ value at which maximum error occurs:

$$
\alpha_{m}=\frac{1+(R \delta-1) e^{R \delta}}{R \delta\left[e^{R \delta}-1\right]}
$$

Numerical evaluations are given in Tables 3 and 4. Table 4 indicates that the $\alpha$ values for the maximum dynamic error are clustered within a narrow band of $0.5 \sim 0.6$.

Table 3. Change of Dynamic Error with $\alpha$

\begin{tabular}{||c|c|c||}
\hline$R(x, y)$ & $\begin{array}{c}E q .(B-14) \\
\delta=0.5, \alpha=0.5\end{array}$ & $\begin{array}{c}\text { Eq. (B-14) } \\
\delta=0.8, \alpha=0.5\end{array}$ \\
\hline 0.1 & 0.0001 & 0.0004 \\
\hline 0.5 & 0.0026 & 0.0105 \\
\hline 1.0 & 0.0102 & 0.0401 \\
\hline 2.0 & 0.0379 & 0.1360 \\
\hline
\end{tabular}

Table 4. Values of $\alpha$ for Maximum Dynamic Error

\begin{tabular}{|c|c|c|c|}
\hline \multirow{2}{*}{$R(x, y)$} & \multicolumn{3}{|c|}{$\alpha_{m}$ in. Eq. (B-15) } \\
\cline { 2 - 4 } & $\delta=0.2$ & $\delta=0.5$ & $\delta=0.8$ \\
\hline 0.1 & 0.5017 & 0.5042 & 0.5067 \\
\hline 0.5 & 0.5083 & 0.5208 & 0.5332 \\
\hline 1.0 & 0.5167 & 0.5415 & 0.5660 \\
\hline 2.0 & 0.5332 & 0.5820 & 0.6280 \\
\hline
\end{tabular}


(c) Change with $F_{1}(x, y)$

Taking the derivative of Eq. (B-12) with respect to $F_{1}(x, y)$, the minimum void fraction, gives

$$
\begin{aligned}
\frac{\partial \Delta \bar{F}(x, y)}{\partial F_{1}(x, y)} & =\frac{1}{R} \frac{\alpha R e^{R F_{1}}+(1-\alpha) R e^{R\left[F_{1}+\delta\right]}}{\alpha e^{R F_{1}}+(1-\alpha) e^{R\left[F_{1}+\delta\right]}}-\alpha-(1-\alpha) \\
& =1-\alpha-1+\alpha \\
& =0 .
\end{aligned}
$$

This result implies that the dynamic error $\Delta \bar{F}(x, y)$ is independent of the minimum void fraction $F_{1}(x, y)$ during a time period $T$. Hence, the error will be the same whether the void fraction fluctuates between $F_{1}(x, y)$ and $F_{2}(x, y)$ as long as the value $\delta(x, y)=F_{2}(x, y)-F_{1}(x, y)$ is constant. Hence Eq. (B-12) can be simplified by setting $F_{1}(x, y)=0$, without changing the dynamic error in anyway. When this is done, the result is,

$$
\left.\partial \Delta \bar{F}(x, y)\right|_{F_{1}=0.0}=\frac{1}{R(x, y)} \ln \left\{\alpha+(1-\alpha) e^{F \delta}\right\}-(1-\alpha) \delta(x, y) .
$$

A numerical evaluation of Eq: (B-16) is given in Table 5.

Table 5. Dynamic Error

\begin{tabular}{|c|c|c|}
\hline$R(x, y)$ & $\begin{array}{c}\text { Eq. (B-16) } \\
\delta=0.5, \alpha=0.5\end{array}$ & $\begin{array}{c}\text { Eq. (B-16) } \\
\delta=0.8, \alpha=0.5\end{array}$ \\
\hline 0.1 & 0.0031 & 0.0080 \\
\hline 0.5 & 0.0156 & 0.0397 \\
\hline 1.0 & 0.0309 & 0.0780 \\
\hline 2.0 & 0.0601 & 0.1454 \\
\hline
\end{tabular}

(d) Change with $\delta(x, y)$

Using Eq. (B-12) or Eq. (B-16), we have

$$
\begin{aligned}
\frac{\partial \Delta \bar{F}(x, y)}{\partial \delta(x, y)} & =\frac{1}{R} \frac{(1-\alpha) R e^{R\left[F_{1}+\delta\right]}}{\alpha e^{R F_{1}}+(1-\alpha) e^{\left.R F_{1}+\delta\right]}}-(1-\alpha) \\
& =\frac{(1-\alpha) e^{R \delta}}{\alpha+(1-\alpha) e^{R \delta}-(1-\alpha) .} \\
\frac{\partial \Delta \bar{F}(x, y)}{\partial \delta(x, y)} & \left.=(1-\alpha) \alpha \frac{e^{R \delta}-1}{\alpha+(1-\alpha) e^{R \delta}}\right] .
\end{aligned}
$$


This expression is positive for all possible values of $\alpha, R(x, y)$, and $\delta(x, y)$. This implies that the error increases as the difference $\delta(x, y)$ between $F_{1}(x, y)$ and $F_{2}(x, y)$ increases. The maximum error occurs when $F_{1}(x, y)=0.0$ and $F_{2}(x, y)=1.0$, that is, when maximum void fluctuation occurs. This condition is applicable to plastic discs with fulldepth holes. When $\delta(x, y)=1.0$ is introduced into Eq. $(B-16)$, the result is as follows and a numerical evaluation of the result is shown in Table 6.

$$
\Delta \bar{F}(x, y)=\frac{1}{R(x, y)} \ln \left\{\alpha+(1-\alpha) e^{R(x, y)}\right\}-(1-\alpha) .
$$

Table 6. Change of Dynamic Error with $\delta(x, y)$

\begin{tabular}{|c|c|c|}
\hline$R(x, y)$ & $\begin{array}{c}\text { Eq. }(B-17) \\
\delta=0.5, \alpha=0.5\end{array}$ & $\begin{array}{c}\text { Eq. }(B-17) \\
\delta=0.8, \alpha=0.5\end{array}$ \\
\hline 0.1 & 0.0125 & 0.0200 \\
\hline 0.5 & 0.0622 & 0.0987 \\
\hline 1.0 & 0.1225 & 0.1900 \\
\hline 2.0 & 0.2311 & 0.3320 \\
\hline
\end{tabular}

(e) Maximum Error

Equation (B-18) can be used to find the maximum dynamic error when the maximum value of $\alpha, \alpha_{m}$ given in Eq. (B-15), is introduced into it.

When $\delta(x, y)=1.0, \alpha_{m}$ given in Eq. (B-15) now becomes

$$
\left.\alpha_{m}\right|_{\delta=1.0}=\frac{1+[R(x, y)-1] e^{R(x, y)}}{R(x, y)\left[e^{R(x, y)}-1\right]}
$$

Substituting Eq. (B-19) into Eq. (B-18) produces "maximum" dynamic error as a function of $R(x, y)$ only:

$$
\begin{aligned}
\Delta \bar{F}_{\max }(x, y)=\frac{1}{R(x, y)} & \ln \left\{\frac{1+[R-1] e^{R}}{R\left[e^{R}-1\right]}+\left[1-\frac{1+(R-1) e^{R}}{R\left(e^{R}-1\right)}\right] e^{R}\right\} \\
& -\left[1-\frac{1+(R-1) e^{R}}{R\left(e^{R}-1\right)}\right] .
\end{aligned}
$$

The maximum dynamic error increases almost linearly with the path thickness $R(x, y)$ as illustrated in Table 7 and Figure 3. 
Table 7. Maximum Dynamic Error versus $R(x, y)$

\begin{tabular}{|c|c|c|}
\hline$R(x, y)$ & $\begin{array}{c}\text { Eq. }(B-19) \\
\alpha_{m}\end{array}$ & $\begin{array}{c}E q .(B-20) \\
\Delta \bar{F}_{\max }\end{array}$ \\
\hline 0.1 & 0.5083 & 0.0125 \\
\hline 0.5 & 0.5415 & 0.0623 \\
\hline 1.0 & 0.5820 & 0.1233 \\
\hline 1.5 & 0.6206 & 0.1819 \\
\hline 2.0 & 0.6565 & 0.2372 \\
\hline 3.0 & 0.7191 & 0.3358 \\
\hline
\end{tabular}

For a plastic disc with holes, $\mu_{v}=0.0 \mathrm{~cm}^{-1}, \mu_{w} \approx 3.0 \mathrm{~cm}^{-1}$ and $T(x, y) \approx$ $0.22 \mathrm{~cm}$, resulting in $R(x, y)=0.66$. We can see in Figure 3 that the maximum possible dynamic error is 0.08 in vapor fractions, which was predicted earlier in Figure 2. It should be noted that this number is an upper limit and does not represent the conditions encountered in the acrylic disc measurements.

(f) Hiah Pressure Hiah Temperature Conditions

As was discussed in Section III.1.1, the maximum dymamic error derived in this section is an overestimate for a pressurized hot conduit because the attenuation coefficient of vapor (steam), $\mu_{v}$, was assumed zero in Equation (B-12). For a pressurized hot conduit, the attenuation coefficient of water, $\mu_{w}$, must be replaced by $\left(\mu_{w}-\mu_{w}\right)$ so that $R(x, y)$ in Equation (B-20) becomes $\left(\mu_{w}-\mu_{v}\right) T(x, y)$. The maximum dynamic error in vapor fractions for a conduit where $\mu_{v}=0.5 \mathrm{~cm}^{-1}$ and $\mu_{w}=3.0 \mathrm{~cm}^{-1}$ was calculated for various conduit thicknesses and shown in Figure 3. The dynamic error in such a conduit is reduced by approximately 15 percent compared to conditions at standard pressure and temperature.

\section{III.2 Collision Probability Method}

The Collision Probability Method, developed in Reference 6, analytically determines the scattering component of transmitted neutron beam in the data reduction process and removes it from the neutron beam intensity measured in experiments. In this method, a collision probability of each incident beam is calculated by applying a hydrogen scattering kernel to scattered neutrons that interact with water, leave the incident beam, but eventualiy show up elsewhere in the experiment. The uncollided beam intensity is calculated and used to determine the vapor fraction distribution in the fluid. In this section, the dynamic error resulting from the Collision Probability Method is determined following the same procedure used in the binomial vapor fraction case (Section III.1.1).

The vapor fraction along the beam path through the point $(x, y)$ at a test section is given in terms of the collision probability $P(x, y, t)$ at time $t$ as follows (Reference 7 ): 


$$
F(x, y, t)=\frac{1+\frac{\ln [1-P(x, y, t)]}{\sigma N_{w} T(x, y)}}{1-\left(N / N_{w}\right)},
$$

where

$$
\begin{array}{ll}
\sigma & =\text { microscopic total cross section of hydrogen, } \\
N_{w} & =\text { hydrogen number density in vapor, } \\
N_{w} & =\text { hydrogen number density in water, and } \\
T(x, y) & =\text { the flow path thickness through }(x, y) .
\end{array}
$$

Define the following terms:

$$
\begin{array}{ll}
C_{0} & =\left[1-N_{w} / N_{w}\right]^{-1} \\
R(x, y) & =\sigma N_{w} T(x, y) \\
N(x, y, t) & =1-P(x, y, t) \\
& =\text { non-collision probability }
\end{array}
$$

Equation (B-21) is then written by

$$
\begin{aligned}
F(x, y, t) & =C_{0}\left[1+\frac{\ln N(x, y, t)}{R(x, y)}\right] \\
& =C_{0}+C_{1} \ln N(x, y, t),
\end{aligned}
$$

where $C_{1}=C_{d} / R(x, y)$.

Because of the one-to-one relationship between the non-collision probability $N(x, y, t)$ and the vapor fraction $F(x, y, t)$ indicated by Eq. (B-22), the binomial pattern of $F(x, y, t)$ introduced in Section III.1.1 can also be applied to $N(x, y, t)$.

The vapor fraction determined by the Count-Mode measure is given by

$$
\begin{aligned}
\bar{F}_{c}(x, y) & =C_{0}+C_{1} \ln \left[\frac{1}{n} \sum_{j=1}^{n} N_{j}(x, y)\right] \\
& =C_{0}+C_{1} \ln \left[\alpha N_{1}+(1-\alpha) N_{2}\right]
\end{aligned}
$$

where $N_{j}(x, y)$ represents the value of $N(x, y, t)$ during $\Delta t$ from $t_{j}$ to $t_{j}+\Delta t$, and $N_{1}$ and $N_{2}$ are the minimum and maximum values of $N(x, y, t)$, respectively. The fraction of the time period $\alpha$ is the same as defined in Section III.1.1.

The Exact Measure will produce an exact vapor fraction as follows:

$$
\begin{aligned}
\bar{F}(x, y) & =c_{0}+c_{1} \ln \left[\frac{1}{n} \prod_{j=1}^{n} N_{j}(x, y)\right]^{\frac{1}{n}} \\
& =C_{0}+C_{1} \ln \left[N_{1}^{\alpha} N_{2}^{1-\alpha}\right] .
\end{aligned}
$$


Subtracting Eq. (B-24) from Eq. (B-23) yields the dynamic error:

$$
\begin{aligned}
\Delta \bar{F}(x, y)= & C_{1}\left\{\ln \left[\alpha N_{1}+(1-\alpha) N_{2}\right]-\right. \\
& \left.\alpha \ln N_{1}-(1-\alpha) \ln N_{2}\right\}
\end{aligned}
$$

From Eq. (B-22), we have a relationship between $N(x, y)$ and $F(x, y)$ at any time t:

$$
N(x, y)=\exp \left\{R(x, y)\left[F(x, y) / C_{0}-1\right]\right\}
$$

That is,

$$
N_{1}=\exp \left(F_{1} / C_{1}-R\right)
$$

and

$$
N_{2}=\exp \left(F_{2} / C_{1}-R\right)
$$

Using these relationships, the dynamic error becomes

$$
\begin{aligned}
\Delta \bar{F}(x, y) & =C_{1} \ln \left[\alpha e^{F_{1} / C_{1}}+(1-\alpha) e^{\left(F_{1}+\delta\right) / C_{1}}\right] \\
& -\alpha F_{1}-(1-\alpha)\left(F_{1}+\delta\right) .
\end{aligned}
$$

Equation (B-27) is identical to Equation (B-20), indicating that the dynamic error is invariant when a scattering correction technique based on the Collision Probability Method is applied to the Count-Mode measurement. In Equation (B-27), the identity $F_{2}(x, y)=F_{1}(x, y)+\delta(x, y)$ has been used as in the Exponential Attenuation Method.

Since the general expression of the dynamic error with the Collision Probability Method is unchanged from that with the Exponential Attenuation Method, the characteristics of the dynamic error and its maximum value discussed in Section III.1.2 are unaffected by the use of the Collision Probability Method.

\section{III.3 Dynamic Error from Linear Vapor Fraction}

We have conducted a dynamic error analysis in Section III.1.1 utilizing a binomial pattern of vapor fraction distribution, which is considered to be an extreme case that can result in the most severe dynamic errors. Only the lowest and highest values of the vapor fraction were assumed present during a measurement period, precluding intermediate vapor fractions between the limits. As an intermediate case, we examine a condition where the vapor fraction at a spatial element tends to be smooth and continuous rather than abrupt and discontinuous (as a function of time). A simplistic, smooth and continuous fluctuation of vapor fraction as a function of time can be modeled by a linear function. A linear vapor fraction distribution during a measurement period and associated dynamic error are discussed in this section. Because of the identical dymamic error resulting from both the Exponential Attenuation Method and the Collision Probability Method when a binomial pattern was considered, only the Exponential Attenuation Method is being considered in this section. 


\section{III.3.1 Linear Vapor Fraction}

Consider a case where $F_{1}(x, y)$ is the lowest vapor fraction and $F_{1}(x, y)+$ $\delta(x, y)$ is the highest vapor fraction at $(x, y)$ during a measurement period of $T$. If intermediate vapor fractions are represented by a linear function of time $t$, we have a vapor fraction at $t$ as follows:

$$
F(x, y, t)=F_{1}(x, y)+\frac{\delta(x, y)}{T} t
$$

It has been assumed in Eq. (B-28) that $F_{1}(x, y)$ occurs at $t=0$ and the highest vapor fraction at the end of the time period. The assumption has been made just for convenience, and indeed it can represent any linear fluctuation case where the time-integration of individual values, not the sequence of individual values, is of interest.

In Count-Mode measure, the time-averaged vapor fraction was defined by Eq. (B-2):

$$
\overline{F_{c}}(x, y)=c_{1} \ln \bar{V}(x, y)+c_{2} .
$$

The constants $C_{1}$ and $C_{2}$ are the same as defined in Section III.1. Since the intensity of transmitted beam $V(x, y, t)$ is

$$
V(x, y, t)=e^{-\left[C_{2}-F(x, y, t)\right] / C_{1}}
$$

its time-averaged value $\bar{V}(x, y)$ becomes

$$
\begin{aligned}
\bar{V}(x, y) & =\frac{1}{T} \int_{0}^{T} e^{-\left[C_{2}-F_{1}(x, y)-\frac{1}{T} \delta(x, y) t\right] / C_{1}} d t \\
& =\frac{1}{T} e^{-\left[C_{2}-F_{1} / C_{1}\right.} \int_{0}^{T} e^{\frac{\delta t}{T C_{1}}} d t \\
& =\frac{C_{1}}{\delta} e^{-\left[C_{2}-F_{1} y C_{1}\right.} \cdot\left(e^{\delta / C_{1}}-1\right) .
\end{aligned}
$$

Substituting Eq. (B-30) into Eq. (B-29) yields

$$
\bar{F}_{c}(x, y)=C_{1} \ln \left[\frac{C_{1}}{\delta(x, y)}\left\{e^{\delta(x, y) / C_{1}}-1\right\}\right]+F_{1}(x, y) .
$$

In Exact Measure, we can directly integrate Eq. (B-28) to determine the exact time-averaged vapor fraction $\bar{F}(x, y)$ :

$$
\begin{aligned}
\bar{F}(x, y) & =\frac{1}{T} \int_{0}^{T}\left[F_{1}(x, y)+\frac{\delta(x, y)}{T} t\right] d t \\
& =F_{1}(x, y)+\frac{1}{2} \delta(x, y) .
\end{aligned}
$$


Therefore, the dynamic error can be calculated by subtracting Eq. (B-32) from Eq. (B-31):

$$
\begin{aligned}
\Delta \bar{F}(x, y) & =\bar{F}_{c}(x, y)-\bar{F}(x, y) \\
& =\frac{1}{R(x, y)} \ln \left[\frac{1}{R(x, y) \delta(x, y)}\left\{e^{R(x, y) \delta(x, y)}-1\right\}\right]-\frac{\delta(x, y)}{2},
\end{aligned}
$$

where $R(x, y)=\mu_{w} T(x, y)$, and $\mu_{v} * \mu_{w}$ was assumed.

Equation (B-33) indicates that the dynamic error, under a linear vapor fraction variation with respect to time, is independent of $F_{1}(x, y)$, the lowest vapor fraction, but dependent on the relative flow path thickness, $R(x, y)$, and the extent of vapor fraction variation, $\delta(x, y)$.

\section{III.3.2 Maximum Dynamic Error Estimate - Linear Vapor Fraction}

To observe how each variable in Eq. (B-33) affect the dynamic error, the derivatives of the equation taken with respect to each variable are examined in this section. Finding and using the limiting value of each variable, a hypothetical maximum permissible dynamic error is then determined.

(a) Change with $u_{n} T(x, y)$

Taking the derivative of Eq. (B-33) with respect to $R(x, y)$ (i.e., $\mu_{w} T(x, y)$ ) yields

$$
\begin{aligned}
\frac{\partial \Delta \bar{F}(x, y)}{\partial R(x, y)} & =\frac{-1}{R^{2}}\left[\ln \left(e^{R \delta}-1\right)-\ln R \delta\right]+\frac{\delta}{R} \frac{e^{R \delta}}{e^{R \delta}-1}-\frac{1}{R^{2}} \\
& =-\frac{1}{R^{2}}\left[\ln \left\{\frac{e^{R \delta}-1}{R \delta}\right\}+1\right]+\frac{\delta}{R} \frac{e^{R \delta}}{e^{R \delta}-1} .
\end{aligned}
$$

A numerical evaluation of Eq. (B-34), shown in Table 8 indicates that $\partial \Delta \bar{F} / \partial R$ is greater than zero for $R(x, y)$ and $\delta(x, y)$ within the range of interest. Relative to the binomial vapor fraction case (Table 2), $\partial \Delta \bar{F} / \partial R$ is less sensitive to $R(x, y)$ and reduced by a factor of three. 
Table 8. Change of Dynamic Error with Flow Path Thickness (Linear Vapor Fraction Model)

\begin{tabular}{|c|c|c|c|c|}
\hline \multirow{2}{*}{$R(x, y)$} & \multicolumn{4}{|c|}{ Eq. (B-34) } \\
\cline { 2 - 5 } & $\delta=0.2$ & $\delta=0.5$ & $\delta=0.8$ & $\delta=1.0$ \\
\hline 0.1 & 0.00167 & 0.01042 & 0.0267 & 0.0417 \\
\hline 0.5 & 0.00167 & 0.01040 & 0.0266 & 0.0414 \\
\hline 1.0 & 0.00167 & 0.01035 & 0.0263 & 0.0407 \\
\hline 2.0 & 0.00166 & 0.01016 & 0.0251 & 0.0379 \\
\hline
\end{tabular}

(b) Change with $\delta(x, y)$

Taking the derivative of Eq. (B-33) with respect to $\delta(x, y)$ produces

$$
\frac{\partial \Delta \bar{F}(x, y)}{\partial \delta(x, y)}=\frac{e^{R \delta}}{e^{R \delta}-1}-\frac{1}{R \delta}-\frac{1}{2} .
$$

A numerical evaluation shown in Table 9 indicates that $\partial \Delta \bar{F}(x, y) / 2 \delta$ is positive for $R(x, y)$ and $\delta(x, y)$ values of interest, and for a given $R(x, y)$ it increases with $\delta(x, y)$, the extent of fluctuation in the vapor fraction. This implies that the maximum dynamic error occurs when the $\delta(x, y)$ value reaches its maximum permissible value, unity. This is the same as the binomial vapor fraction case. However, the change of $\bar{\Delta} F(x, y)$ with $\delta(x, y)$ is smaller than the binomial case (Table 6 ) by a factor of three.

Table 9. Change of Dynamic Error with $\delta(x, y)$ - Linear Model

\begin{tabular}{||c|c|c|c|c|}
\hline \multirow{2}{*}{$R(x, y)$} & \multicolumn{4}{|c|}{ Eq. $(B-35)$} \\
\cline { 2 - 5 } & $\delta=0.2$ & $\delta=0.5$ & $\delta=0.8$ & $\delta=1.0$ \\
\hline 0.1 & 0.0017 & 0.0042 & 0.0067 & 0.0083 \\
\hline 0.5 & 0.0083 & 0.0208 & 0.0332 & 0.0415 \\
\hline 1.0 & 0.0167 & 0.0415 & 0.0660 & 0.0820 \\
\hline 2.0 & 0.0332 & 0.0820 & 0.1280 & 0.1565 \\
\hline
\end{tabular}

(c) Maximum Error

Equation (B-33) can be used to determine the maximum dynamic error when the maximum permissible value of $\delta(x, y)$, unity, is introduced into it. Shown in Table 10 are dynamic errors as a function of $\delta(x, y)$ for several flow path dimensions of interest. The last column shows the maximum permissible error in the worst case of fluctuation in vapor fractions ranging from zero to unity. 
Table 10. Maximum Dynamic Error versus Flow Path Thickness - Linear Model

\begin{tabular}{|c|c|c|c|c|}
\hline \multirow{2}{*}{$R(x, y)$} & \multicolumn{4}{|c|}{ Eq. (B-33) } \\
\cline { 2 - 5 } & $\delta=0.2$ & $\delta=0.5$ & $\delta=0.8$ & $\delta=1.0$ \\
\hline 0.1 & 0.0002 & 0.0010 & 0.0027 & 0.0042 \\
\hline 0.5 & 0.0008 & 0.0052 & 0.0133 & 0.0208 \\
\hline 1.0 & 0.0017 & 0.0104 & 0.0265 & 0.0413 \\
\hline 2.0 & 0.0033 & 0.0207 & 0.0522 & 0.0807 \\
\hline
\end{tabular}

Relative to the binomial vapor fraction case shown in Section III.1.2 (Figure 3), the linear vapor fraction model results in smaller dynamic errors by a factor of three for a wide range of $R(x, y)$ as illustrated in Figure 4. For a two-phase flow of interest, $R(x, y)=0.66$. This yields a maximum possible dynamic error of 0.027 in vapor fraction. It should be noted that this error is an upper limit under the worst case assumption of unity $\delta(x, y)$.

\section{III.3.3 Estimate of Dynamic Error for Acrylic Disc Measurements.}

In Reference 3, vapor-water mixtures were simulated using acrylic discs containing various size holes which appear to the neutrons as vapor regions. Fluid flow was simulated by rotating the discs. Time averaged void fractions were determined using the Count-Mode technique and compared with the exact average disc void fraction deduced from the known hole sizes. Absolute differences of a few percentage points between the measured and true void fractions were observed and judged, in Reference 3 , to be due to uncertainties in measurements and dimensional tolerances of the test device. The dynamic error, however, was not considered. When the disc containing holes of 0.5 inch in diameter (drilled every 10 degrees apart) rotates at the speed of $30 \mathrm{rpm}$, each time subinterval of 1/30 sec covers 6 degrees of the disc. In this case, the minimum and maximum void fractions at a stationary point $(x, y)$ on the circumferential line connecting the centers of the innermost holes would be 0.07 and 0.74 , respectively, resulting in the fluctuation in the void fraction of $\delta(x, y)=0.67$. If we assume that the temporal distribution of the void fraction at the point $(x, y)$ is approximated by the linear function of time defined by Equation (B-28), then the dynamic error in the void fraction will be 0.012 when $R(x, y)$ $=0.66$ is used in Equation $(B-33)$ for the acrylic disc.

\section{N. SUMMARY AND CONCLUSIONS}

A neutron radiography system has been developed and is being used for real-time visualization of dynamic behavior as well as time-averaged measurements of spatial vapor fraction distributions for two-phase fluids in opaque conduits. An analysis of both the static error and dymamic error was conducted to help quantify the achievable maximum errors and also to provide insight into the systematic errors associated with the neutron radiography technique developed for vapor fraction measurements.

The static error is a measure of the statistical uncertainty interval in the vapor fraction. It is determined by the propagation of uncertainty intervals of measured intensities of neutron beams. It has been found that the maximum static error 
occurs at zero vapor fraction and is slightly sensitive to the flow path thickness within the range of interest. For flow path thicknesses ranging from $0.3 \mathrm{~cm}$ to $1.3 \mathrm{~cm}$, the maximum error in vapor fraction is 0.02 . This result is based on the neutron flux level $2 \times 10^{6} \mathrm{n} / \mathrm{cm}^{2} / \mathrm{s}$. With increased source beam intensity the error can be reduced by a factor proportional to the square root of the gain in the source beam intensity.

The dynamic error in the vapor fraction is due to the inherent time-dependence of the measured data when the data is time-averaged by the Count-Mode measure. The dynamic error depends primarily upon and increases with the extent of the fluctuation in the local density of two-phase fluids and the flow path thickness. For the case where the flow path thickness is $0.22 \mathrm{~cm}$, it has been found that the maximum possible dynamic error under the most extreme condition could be as large as 0.08 in vapor fractions. However, this number is a worst case upper limit and significantly larger than what was observed during an actual data acquisition process using the Exact Measure technique in the void fraction measurements of the acrylic discs. The majority of vapor fraction measurements involve less extreme conditions which result in smaller errors. For a less extreme case in which the fluctuation in vapor fractions was modeled as a linear function of time, the maximum dynamic error is approximately 0.03 .

The results of this error analysis lead to the following conclusions: The static error associated with the Penn State reactor facility is relatively small $(0.02)$ and can be reduced by increasing the source beam intensity. In the case of void fraction measurements with the acrylic discs, the dynamic error was determined to be 0.012 . The total error (dynamic plus static) is on the order of $0.026\left(=\left[0.023^{2}+0.012^{2}\right]^{1 / 2}\right)$. For measurements performed at high temperatures and pressures, the dynamic error would be reduced as compared to measurements at standard pressure and temperature. This is due to the non-zero attenuation coefficient of the vapor (steam). Using the Exact Measure technique minimizes the potential dynamic error that is caused by the Count-Mode measure. To do this, the logarithm of the intensity of the neutron beam obtained per video image must be taken before time-averaging the data.

\section{ACKNOWLEDGEMENT}

The authors would like to acknowledge Mr. Wayne A. Surdoval, for bringing this problem area to our attention and for his continual interest and help throughout the development effort. 


\section{References:}

1. S. S. Glickstein, H. Joo and W. H. Vance, "Interpreting Neutron Radiographs Via Computer Simulation," Proceedings of the Fourth World Conference on Neutron Radiography, 761770, May 1992.

2. S. S. Glickstein, W. H. Vance and H. Joo, "Void Fraction Measurements Using Neutron Radiography," Transaction of the ANS/ENS 1992 International Conference, November 1992; Nuc. Sci. \& Eng. 121 153-161 (1995)

3. S. S. Glickstein, H. Joo, W. H. Vance and J. H. Murphy, "Void Fraction Measurements of Acrylic Discs Via Neutron Radiography," Iransaction of the ANS 1994 Annual Meeting, June 1994.

4. S. S. Glickstein, J. H. Murphy and R. B. Hammond, "Void Fraction Measurements in a Steam Water Duct at Atmospheric Pressures Using Neutron Radiography," ASME Conference on Flow Visualization and Image Processing of Multiphase Systems," August 1995.

5. T. J. Honan and R. T. Lahey, Jr., The Measurement of Phase Separation in Wyes and Tees," NUREG/CR-0557, December 1978.

6. S. J. Kine and F. A. McClintock, "Describing Uncertainties in Single Sample Experiments," Trans. ASME, January 1953.

7. H. Joo and S. S. Glickstein, "A Scattering Probability Method for Vapor Fraction Measurements by Neutron Radiography," Third International Topical Meeting on Neutron Radiography, March 16-19, 1998.

8. G. F. Knoll, "Radiation Detection and Measurement," John Wiley \& Sons, New York, 1979.

9. J. L. Bogdanoff and F. Kozin, "Probabilistic Models of Cumulative Damage," John Wiley \& Sons, New York, 1985.

10. M. Verat, H. Rougeot and B. Driard, "Neutron Image Intensifier Tubes," Neutron Radiography by J. P. Barton and P. von der Hardt (eds.) 601-607, ECSC, EEC, EAEC, Brussels and Luxembourg, 1983.

11. D. E. Hughes, "The Development of Real Time Neutron Radiography at the Penn State Breazeale Reactor," M. S. Thesis, Pennsylvania State University, December 1986. 
Figure 1

Maximum Static Error Versus Flow Path Thickness*

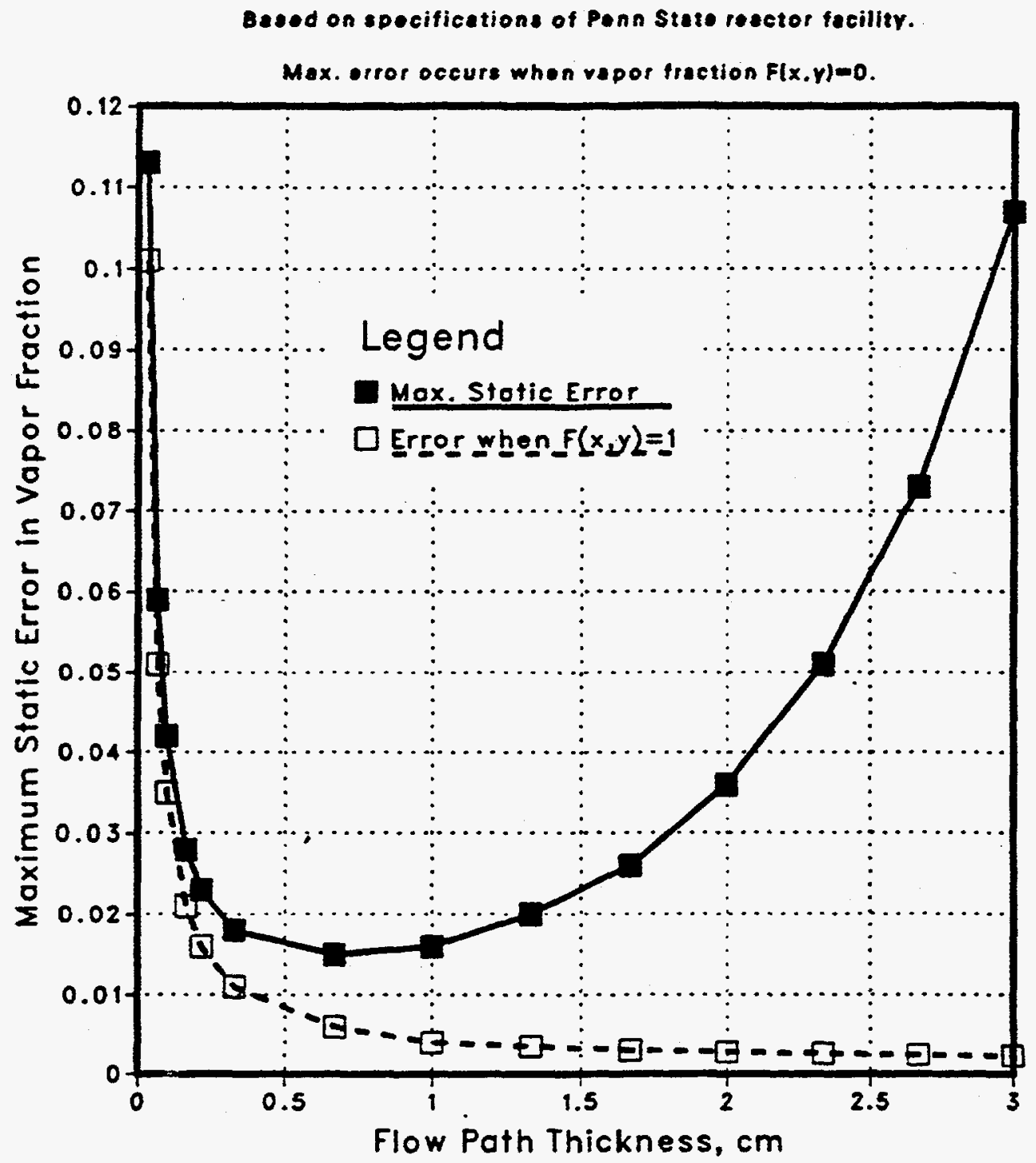

*The attenuation coefficient of water of $3.0 \mathrm{~cm}^{-1}$ is used. 
Figure 2

Dynamic Error in Void Fractions for an Acrylic Disc with Holes*

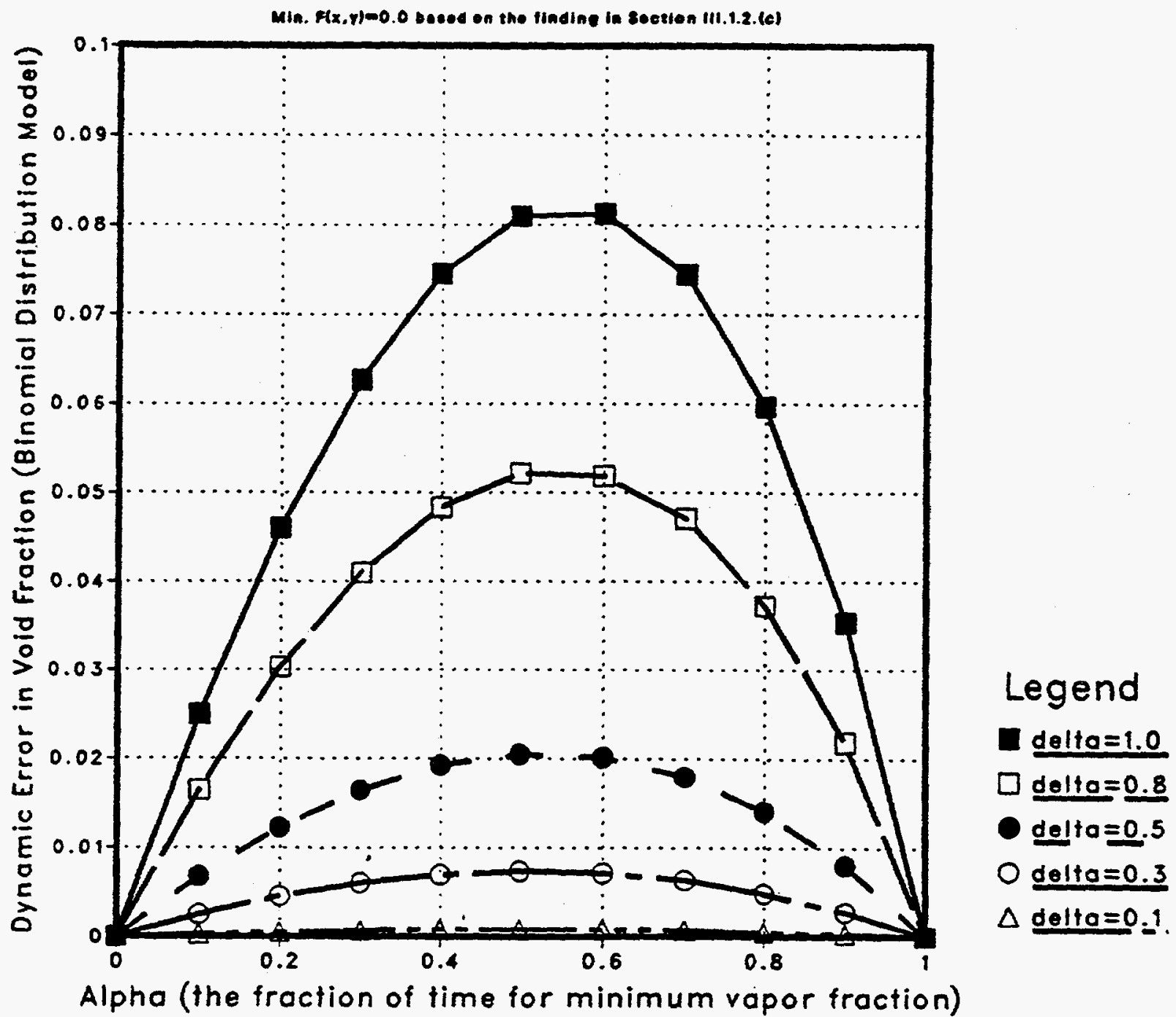

Delta $=$ fluctuation in local void fractions during a measurement period - maximum void fraction - minimum void fraction

- Attenuation coefficient of the disc $=3.0 \mathrm{~cm}^{-1}$ Thickness of the disc $=0.22 \mathrm{~cm}$ 
Figure 3

Maximum Dynamic Error Versus Flow Path Thickness*

Napor Fraction Pattern $=$ Binomial Model

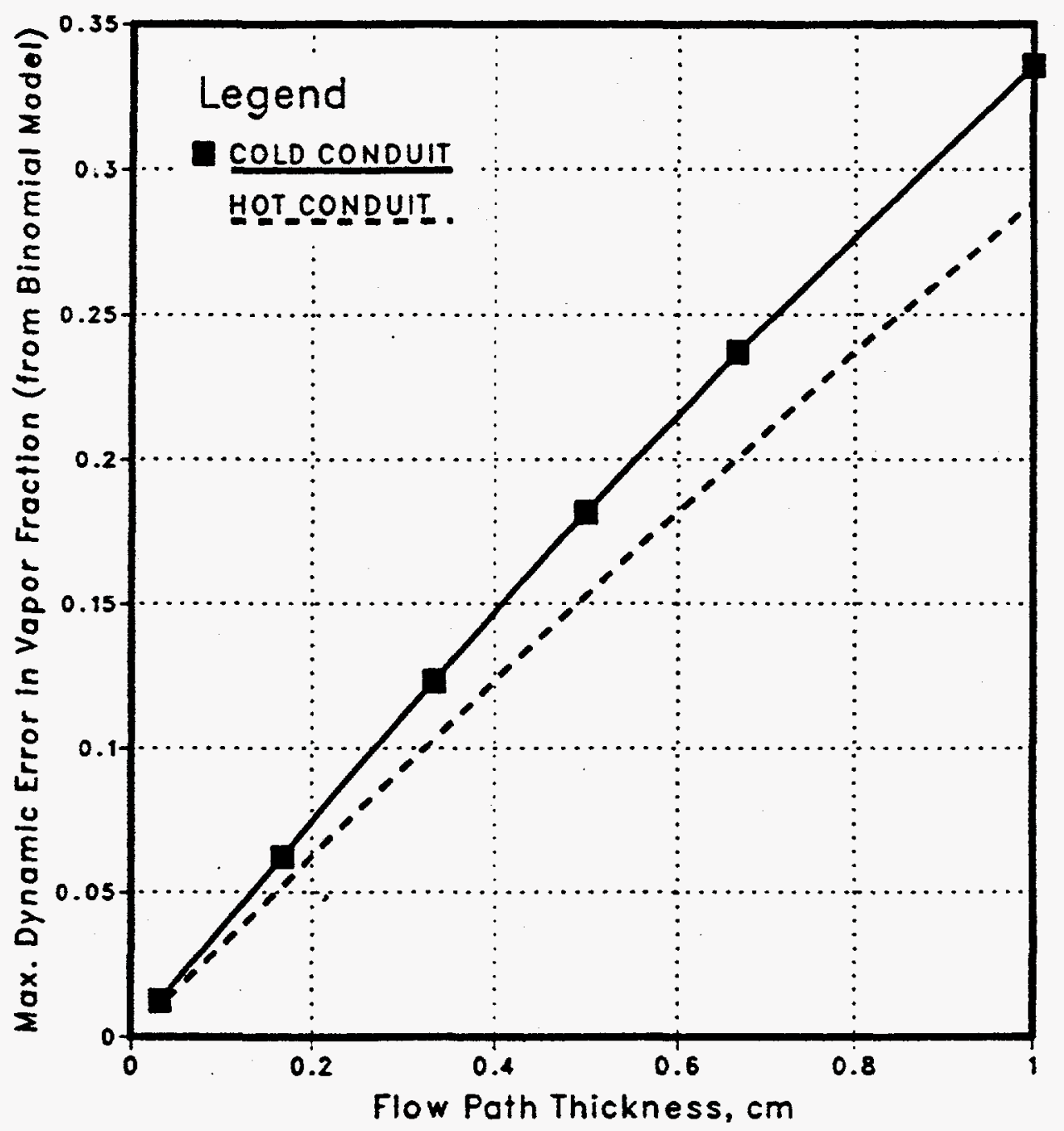

Alpha = optimum value for the maximum error

Deita $=1.0$ for the worst case

*The attenuation coefficient of water of $3.0 \mathrm{~cm}^{-1}$ is used. The attenuation coefficients of vapor at the cold and hot conduits are $0.0 \mathrm{~cm}^{-1}$ and $0.5 \mathrm{~cm}^{-1}$, respectively. 
Figure 4

Maximum Dynamic Error Versus Flow Path Thickness*

Napor Fraction Pattern - Linear Model)

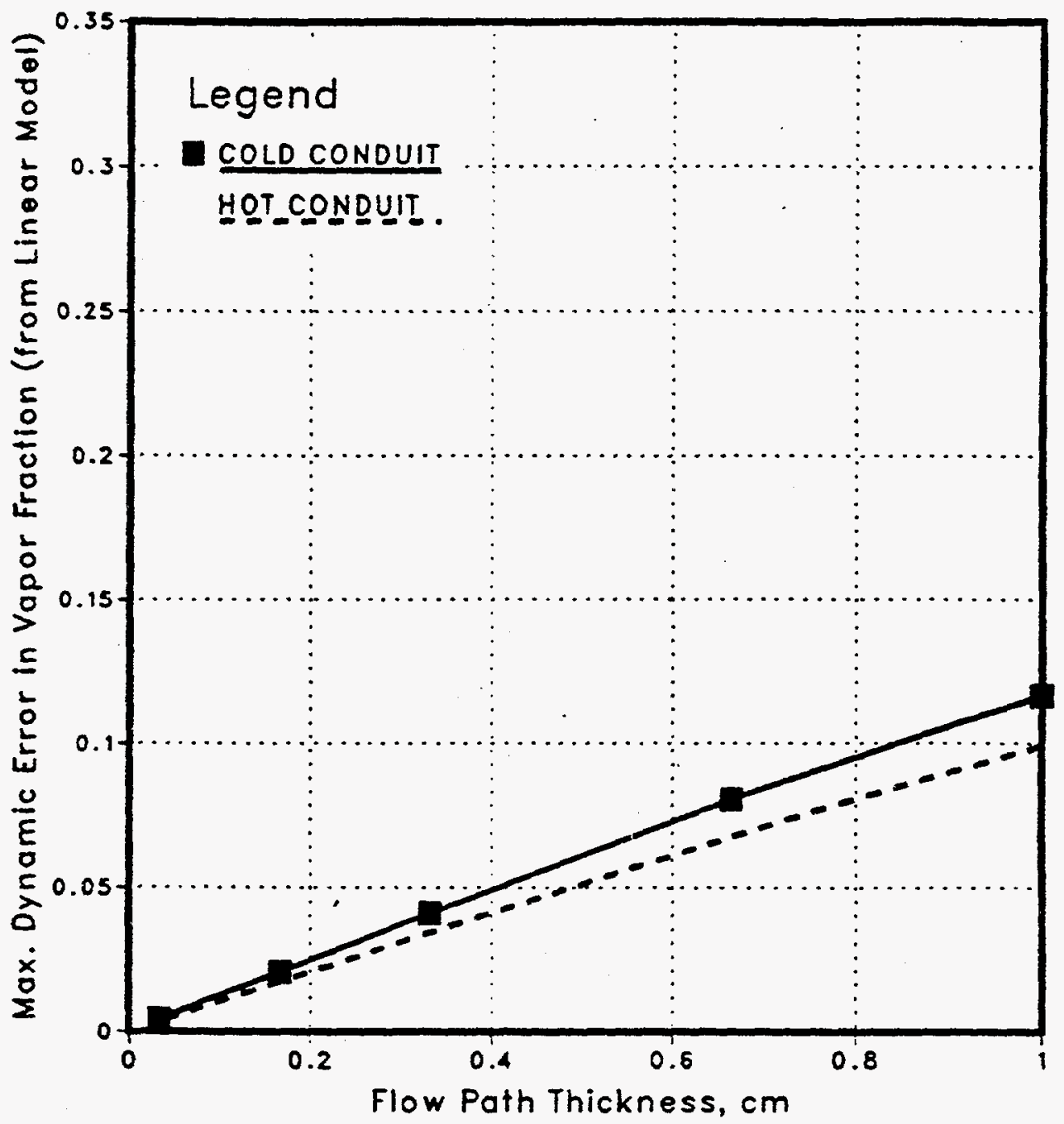

Alpha $=$ optimum value for the maximum error

Delta $=1.0$ for the worst case

*The attenuation coefficient of water of $3.0 \mathrm{~cm}^{-1}$ is used. The attenuation coefficients of vapor at the cold and hot conduits are $0.0 \mathrm{~cm}^{-1}$ and $0.5 \mathrm{~cm}^{-1}$, respectively. 